\title{
Neu berufene Professorinnen und Professoren
}

\section{Univ.-Prof. Dr. Orhan Aktaş}

Im April 2008 wurde Orhan Aktaş auf die Professur für Molekulare Neurologie an die Neurologische Klinik des Universitätsklinikums Düsseldorf berufen.

Er wurde 1972 in Mersin in der Türkei geboren und studierte als Stipendiat der Konrad-Adenauer-Stiftung von 1992 bis 1999 Humanmedizin an der Ruhr-Universität Bochum sowie an der Université Louis Pasteur in Straßburg. Im Jahr 2000 wurde er in Bochum mit der Arbeit Einflu $\beta$ von Copolymer-1 auf die Apoptoserate und die Zytokinsynthese von Lymphozyten bei Patienten mit schubförmig remittierender Multipler Sklerose in stabiler Krankheitsphase und bei gesunden Individuen promoviert.

Seine Weiterbildung zum Facharzt für Neurologie absolvierte er an der Charité in Berlin, wo er ab 1999 als

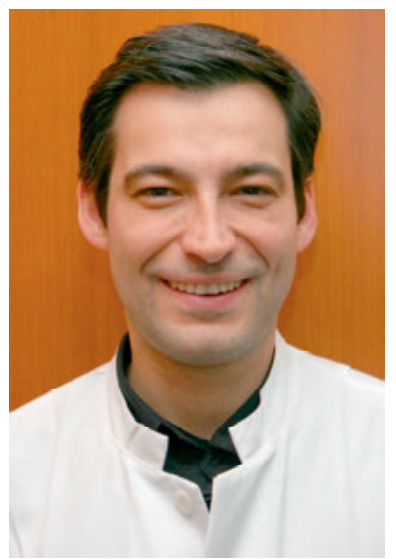
wissenschaftlicher Assistent an der Klinik und Poliklinik für Neurologie arbeitete und im Verlauf an das neu gegründete Institut für Neuroimmunologie wechselte. Im Jahr 2001 erfolgte ein Forschungsaufenthalt als visiting scientist im Department of Experimental Pathology der Hadassah Medical School an der Hebrew University in Jerusalem. Im Jahr 2007 erfolgte die Facharztanerkennung für Neurologie. Im Anschluss wurde er stellvertretender Leiter sowie Oberarzt an der Cecilie-Vogt-Klinik für Molekulare Neurologie der Charité - Universitätsmedizin Berlin. Er habilitierte sich im selben Jahr über die Mechanismen entzündlicher Neurodegeneration bei autoimmuner Demyelinisierung des zentralen Nervensystems und erhielt die Venia legendi für das Fach Neurologie. Seit Oktober 2007 war er Oberarzt an der neu eröffneten Cecilie-Vogt-Klinik für Neurologie im HeliosKlinikum Berlin-Buch/Charité. Er erhielt 2008 die Heisenberg-Professur der Deutschen Forschungsgemeinschaft (DFG) und wurde für die Professur „Regeneration after acute CNS damage" im Berlin-Brandenburger Centrum für Regenerative Therapien (BCRT) primo loco platziert.

Seine translationalen Forschungsprojekte sind um immunologische Mechanismen und die neurobiologische Folgen entzündlich-neurodegenerativer Prozesse zentriert und wurden im Rahmen von zwei Sonderforschungsbereichen der DFG zur Förderung empfohlen. Für seine Arbeiten wurde er mit mehreren Preisen geehrt, darunter mit dem „Teva/Aventis Young Investigator's Award“ der European Charcot Foundation (2001), dem ,Nachwuchspreis“ der Novartis-Stiftung für Therapieforschung (2004), dem „Wyeth Nachwuchswissenschaftler-Preis für Multiple Sklerose Forschung“ (2005) und dem „Multiple Sklerose Forschungspreis“ der Heinrich und Erna Schaufler-Stiftung (2006). Orhan Aktaş ist verheiratet und hat zwei Töchter. 


\section{Univ.-Prof. Dr. Peter Albers}

Am 1. August 2008 wurde Peter Albers auf den Lehrstuhl für Urologie der Universitätskliniken der Heinrich-HeineUniversität Düsseldorf berufen. Er ist seitdem auch Direktor der Klinik für Urologie am Universitätsklinikum Düsseldorf.

1963 in Nürnberg geboren, studierte er von 1982 bis 1988 Humanmedizin und Philosophie zunächst in Aachen und dann Humanmedizin in Mainz. Von 1988 bis 1996 absolvierte er seine urologische Facharztausbildung an den Universitäten Mainz und Bonn (Leitung: Univ.-Prof. Dr. Rudolf Hohenfellner beziehungsweise Univ.-Prof. Dr. Stefan C. Müller). In diese Zeit fiel ein einjähriger Aufenthalt als „Research Fellow“ am Department of Urology der Indiana University, Indianapolis (Leitung: Prof. John P. Donohue) und gleichzeitig die Tätigkeit in der Abteilung Cancer Research der Eli Lilly Headquarters in Indianapolis, Indiana. Forschungsschwerpunkt war die Entwicklung von neuen Prognosefaktoren zur Prädiktion okkulter Metastasen beim nicht-seminomatösen Hodentumor im klinischen Stadium 1. Der Studienaufenthalt wurde durch die Deutsche Forschungsgemeinschaft (DFG) gefördert. Die während des Forschungsaufenthaltes erworbenen Fähigkeiten und Ergebnisse wurden an der Universitätsklinik Bonn sowohl in weiteren Grundlagenprojekten zur Prognosefaktorforschung als auch in klinischen Studien weiter verfolgt. Ab 1996 war Peter Albers für zwei Jahre Oberarzt, ab 1998 leitender Oberarzt an der Urologischen Universitätsklinik Bonn. 1998 folgte ein weiterer klinischer Studienaufenthalt in den Bereichen „Pediatric Urology“ und „Urological Oncology“ am Riley Children's Hospital, Indianapolis, und Children's Hospital of Philadelphia beziehungsweise dem Memorial Sloan Kettering Cancer Center in New York.

1997 habilitierte er sich mit dem Thema Prognosefaktoren im Frühstadium des Hodentumors,im Juli 2002 erfolgte die Ernennung zum außerplanmäßigen Professor für Urologie an der Universität Bonn. Im Jahre 2003 wurde Peter Albers zum Direktor der Urologischen Klinik am Klinikum Kassel GmbH berufen. 2008 erhielt er den Ruf auf den Lehrstuhl für Urologie an der Universitätsklinik Düsseldorf. Während seiner klinischen Ausbildung erwarb er die Zusatzbezeichnung „Spezielle Urologische Chirurgie“ und „Medikamentöse Tumortherapie“.

Peter Albers ist Mitglied in mehreren nationalen und internationalen wissenschaftlichen Gesellschaften. Er wurde mehrfach mit wissenschaftlichen Preisen ausgezeichnet (Maximilian-Nitze-Preis 1999 und 2002). Er ist Vorsitzender der Arbeitsgemeinschaft Urologische Onkologie der Deutschen Krebsgesellschaft und Co-Chairman der Advanced Bladder Cancer Group der GU Group der European Organisation for Research and Treatment of Cancer (EORTC). 


\section{Univ.-Prof. Dr. Wolfgang Janni}

Wolfgang Janni erhielt im März 2008 den Ruf auf den Lehrstuhl der Klinik für Frauenheilkunde der HeinrichHeine-Universität und wurde im Oktober 2008 zum Direktor der Universitätsfrauenklinik ernannt.

1967 wurde er in Augsburg geboren. Er legte 1985 die Allgemeine US-Amerikanische Hochschulreife (High School Diploma) an der Wichita High School East Wichita, Kansas, und 1987 das Abitur am Justus-Liebig-Gymnasium Augsburg ab. Nach der Ableistung des Zivildienstes an den Orthopädischen Fachkliniken der Hessingstiftung Augsburg (1987 bis 1989) begann er 1989 das Studium an der Ludwig-Maximilians-Universität (LMU) München. Seine praktischen Jahre 1994/1995 führten ihn an die I. Frauenklinik der LMU und in die USA an das Hahnemann University Hospital Philadelphia, das Bellevue Hospital

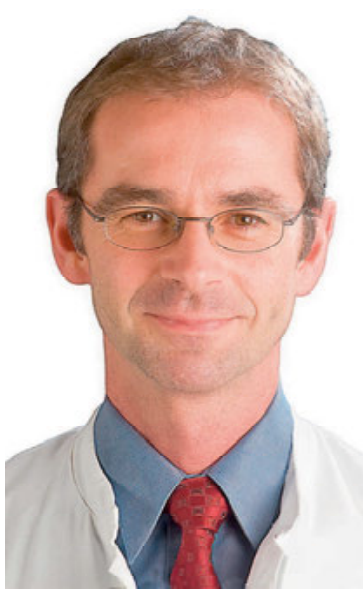
New York City, das University of Michigan Medical Center Ann Arbor sowie das Yale University Medical Center.

Den dritten Abschnitt der Ärztlichen Prüfung legte Wolfgang Janni im November 1995 ab. Von 1995 bis 1997 absolvierte er sein Praktikum an der I. Frauenklinik der LMU. Seine Dissertation hatte das Thema Klinische, prospektive Doppelblindstudie zum Vergleich niedermolekularer Heparine in der orthopädischen Chirurgie.

Von 1997 bis 2002 war er wissenschaftlicher Assistent und Assistenzarzt an der I. Frauenklinik der LMU und habilitierte sich 2003 an der Medizinischen Fakultät mit der Arbeit Die unterschiedliche prognostische Bedeutung hämatogen disseminierter Tumorzellen im Knochenmark zu verschiedenen Zeitpunkten der Brustkrebserkrankung. Einen Ruf auf eine W2-Professur an der Johannes-Gutenberg-Universität Mainz im November 2004 lehnte Wolfgang Janni ab. Er war seit 2002 als klinischer Oberarzt vor allem im Bereich der gynäkologischen Onkologie tätig. Ab Oktober 2005 war er stellvertretender Klinikdirektor der Universitätsfrauenklinik LMU München Innenstadt.

Wolfgang Janni wurde mit mehreren Förderpreisen ausgezeichnet. So erhielt er in den Jahren 2000, 2002, 2004 und 2006 den Merit Award der American Society of Clinical Oncology (ASCO) im Rahmen der jeweiligen Jahrestagungen, im Jahr 2000 für das Abstract „Prognostic Significance of Disseminated Tumor Cells in Bone Marrow of Patients with Primarily Diagnosed Ovarian Cancer“. Ebenfalls im Jahr 2000 wurde er im Rahmen der Jahrestagung der American Association of Cancer Researchers (AACR) mit dem Susan G. Komen Foundation Young Investigator Scholar Award der AACR für das Abstract „Comparative analysis of micrometastasis to the bone marrow and lymph nodes of nodenegative breast-cancer patients receiving no adjuvant therapy“ ausgezeichnet. Sein Beitrag „Persistence of Occult Metastatic Cells in Bone Marrow of Breast Cancer Patients Despite Systemic Adjuvant Treatment“" wurde auf dem 24. Annual San Antonio Breast Cancer Symposium in Texas im Dezember 2001 mit dem Scholars Award ausgezeichnet.

Wolfgang Janni ist Mitglied in verschiedenen nationalen und internationalen Gesellschaften. 


\section{Univ.-Prof. Dr. Carsten Münk}

Carsten Münk, geboren 1964 in Wien, studierte Biologie in Wien und Oldenburg und schrieb seine Diplomarbeit in der Abteilung von Dr. Hansjörg Hauser in der früheren Gesellschaft für Biotechnologische Forschung (GBF), heute Helmholtz-Zentrum für Infektionsforschung, in Braunschweig in dem Forschungsprojekt „Rekombination von retroviralen Vektoren“. In seiner Doktorarbeit in Hamburg am Heinrich-Pette-Institut, Abteilung Univ.Prof. Dr. Wolfram Ostertag, entdeckte er in der Gruppe von Dr. Carol Stocking, dass murine amphotrope Leukämieviren in Mäusen neben der Infektion von Blutzellen auch das Gehirn infizieren können und dort eine spongiforme Enzephalomyelopathie im Kleinhirn und der Medulla oblongata auslösen. Danach arbeitete er als Stipendiat der Deutschen Forschungsgemeinschaft im Labor von Prof. Natha-

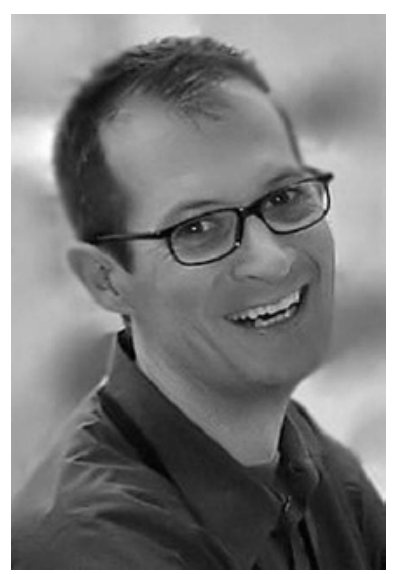
niel Landau am Salk-Institute in La Jolla, Kalifornien, von 1999 bis 2003 am Replikationsblock von HIV-1 in Affen. Es zeigte sich dabei, dass HIV in Affenzellen sehr früh nach der Infektion während der reversen Transkription der viralen RNA gehemmt wird. Neben dieser Restriktion wurde eine weitere spätere Inhibition durch zelluläre ZytidinDeaminasen (APOBEC3) entdeckt.

Ab April 2003 leitete Carsten Münk die Forschergruppe in der Abteilung Medizinische Biotechnologie (Univ.-Prof. Dr. Klaus Cichutek) am Paul-Ehrlich Institut und beschäftigte sich weiter mit antiviralen Zytidin-Deaminasen. Er konnte während dieser Zeit zeigen, dass nicht nur HIV-1 mit seinem viralen Protein Vif, sondern auch Spuma-Retroviren mit deren Protein Bet APOBEC3 Proteine inhibieren. Sowohl Vif wie auch Bet sind essenziell für die virale Replikation. Jüngste Studien zeigen, dass HIV-1 neben dem Vif-Protein weitere, noch unbekannte Mechanismen entwickelt hat, um der antiviralen Aktivität von APOBEC3 auszuweichen.

Seit Juni 2008 arbeitet Carsten Münk als Universitätsprofessor im Rahmen der HeinzAnsmann-Stiftungsprofessur für AIDS-Forschung an der Universitätsklinik Düsseldorf, Klinik für Gastroenterologie, Hepatologie und Infektiologie (Klinikdirektor: Univ.-Prof. Dr. Dieter Häussinger). Die Forschung in Düsseldorf soll verstärkt intrazelluläre HIVAbwehrmechanismen und deren Nutzung zur Entwicklung neuer therapeutischer Medikamente untersuchen. Von großem Interesse wird dabei die Analyse dieser und anderer Faktoren im Krankheitsverlauf von HIV-Patienten der Düsseldorfer HIV-Ambulanz werden. 


\section{Univ.-Prof. Dr. Michael Roden}

Am 2. Juli 2008 wurde Michael Roden zum W3-Professor für Innere Medizin und Direktor der Universitätsklinik für Innere Medizin/Stoffwechselkrankheiten berufen. Am 17. Juli 2008 wurde er zum Direktor des Institutes für Klinische Diabetologie des Deutschen Diabetes-Zentrums der Leibniz Gemeinschaft und Wissenschaftlichen Geschäftsführer der Deutschen Diabetes-Forschungsgesellschaft (DDFG) ernannt.

Er wurde 1961 in Wien geboren und studierte von 1979 bis 1986 Humanmedizin an der Universität Wien. Seine Ausbildung absolvierte er am Pharmakologischen Institut (1986 bis 1988) und an den Universitätskliniken für Innere Medizin I/III (1988 bis 1994) in Wien. Nach der Anerkennung als Facharzt für Innere Medizin 1993 habilitierte er

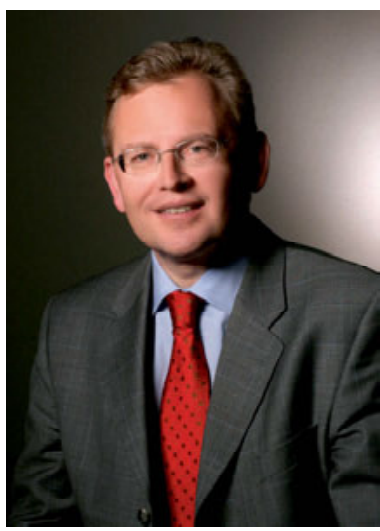
sich 1994 über die Modulation der hepatischen Insulinsensitivität und erhielt die Venia legendi für Innere Medizin.

Als Max-Kade-Fellow untersuchte er in den Jahren 1994 und 1995 am Department of Internal Medicine der Yale University mittels nichtinvasiver Magnetresonanzspektroskopie die Regulation des Leber-Glykogenstoffwechsels und die fettsäureninduzierte Insulinresistenz als Modell der Entstehung von Typ-2-Diabetes.

Michael Roden war von 1995 bis 2003 Oberarzt der Abteilung für Endokrinologie und Stoffwechsel der Universitätsklinik für Innere Medizin III in Wien. Nach Ernennung zum Ao. Univ.-Prof. 1997 erfolgten Facharztanerkennungen für Endokrinologie und Stoffwechsel 1997 und Klinische Pharmakologie 2003. Er war zwischen 2003 und 2008 Vorstand der 1. Medizinischen Abteilung am Hanusch-Krankenhaus, Wien, seit 2005 Direktor des Karl-Landsteiner-Institutes für Endokrinologie/Stoffwechselkrankheiten und seit 2006 Studienkoordinator des Lehrkrankhauses.

Sein wissenschaftliches Interesse gilt besonders dem Energiestoffwechsel des Menschen, klinische Schwerpunkte sind Diabetes, Adipositas und endokrine Hypertonie. Er erhielt mehrfach Auszeichnungen: Ferdinand-Bertram-Preis der Deutschen Diabetes-Gesellschaft 2001, Novartis Award for Innovative Patient Oriented Research 2004, ESCI Award for Excellence in Clinical Sciences 2006, Oskar-Minkowski Prize der European Association for the Study of Diabetes (EASD) 2006, Honorarprofessor für Klinische Endokrinologie der Donau-Universität Krems 2006.

Michael Roden war Präsident der Österreichischen Diabetes-Gesellschaft, Vizepräsident der European Society for Clinical Investigation und Council Member der EASD. Er ist derzeit unter anderem Associate Editor/Board Member von European Journal for Clinical Investigation, Diabetic Medicine und American Journal of Physiology. Er ist verheiratet und hat einen Sohn. 


\section{Prof. Dr. Karin Rothe}

Von 1974 bis 1980 studierte Karin Rothe Medizin an der Universität Leipzig und schloss mit der Diplomarbeit $A l$ tersabhängige Strukturveränderungen der Ligg. Interspinalia an der LWS am Anatomischen Institut der Universität Leipzig ihr Studium ab. Unter dem Ordinariat von Univ.Prof. Dr. Fritz Meissner begann sie 1980 ihre Weiterbildung zum Facharzt für Kinderchirurgie und erhielt 1984 die Anerkennung zum Facharzt für Kinderchirurgie. Nach Abschluss ihrer Promotion mit dem Thema Omphalozele und Laparochisis - retrospektive Studie unter besonderer Berücksichtigung von Risikofaktoren arbeitete Karin Rothe von 1985 bis 1994 als Stationsärztin in der Abteilung für Neugeborenenchirurgie und Notfallmedizin in Leipzig. Von 1987 bis 1990 war sie mit der Forschungsaufgabe

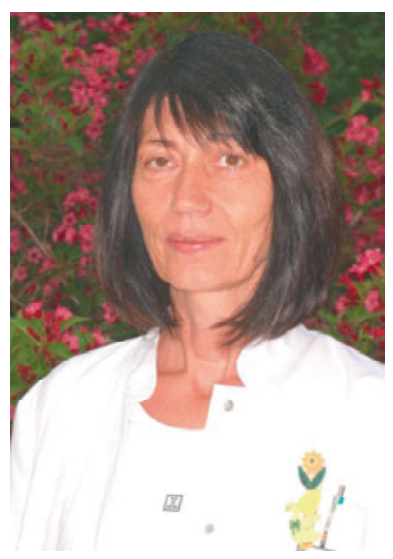
„Die kardiopulmonale Regulation in der perioperativen Phase bei Neugeborenen“ in die Hauptforschungsrichtung (HFR) 20 eingebunden. 1994 folgte die Habilitation mit dem Thema Morbiditätsspezifisches Monitoring in der Kinderchirurgie - Zur Bedeutung biomedizinischer Funktionsgrößen im Neugeborenen- und Säuglingsalter. 1995 erhielt Karin Rothe die Lehrbefugnis für das Fach Kinderchirurgie und übernahm 1996 die Leitung der Abteilung für Neugeborenenchirurgie und Notfallmedizin der Klinik für Kinderchirurgie der Universität Leipzig.

Neben der Neugeborenenchirurgie und allgemeinen Kinderchirurgie gilt Karin Rothes spezielles Interesse der Plastischen Chirurgie einschließlich der Primär- und Sekundärversorgung thermisch verletzter Kinder. Nach einem Studienaufenthalt am Shriners Burns Institut in Galveston, Texas (bei Prof. David Herndon) konnten in Zusammenarbeit mit der Funktionsabteilung Endokrinologie der Universitätskinderklinik Leipzig (Univ.-Prof. Dr. Eberhard Keller) hochdosierte Wachstumshormonbehandlungen zur Beeinflussung der Katabolie und zur schnelleren Regeneration der Spenderhautareale bei den eigenen Patienten erfolgreich durchgeführt werden. Neben ihrer klinischen Tätigkeit war sie intensiv in die Studierendenbetreuung eingebunden. Als Lehrbeauftragte der Klinik organisierte sie die Aus- und Weiterbildung von Ärzten im Praktikum, Studierenden im Praktischen Jahr und Famulanten. Seit 2004 ist sie Vorsitzende der Akademie für Kinderchirurgie der Deutschen Gesellschaft für Kinderchirurgie.

Seit 1. Mai 2008 leitet Karin Rothe den Funktionsbereich Kinderchirurgie der Klinik für Allgemein-, Viszeral- und Kinderchirurgie der Heinrich-Heine-Universität Düsseldorf. Durch die Vielfalt spezialisierter Fachabteilungen im Kinderzentrum kann ein breites Spektrum hochqualifizierter Kinderchirurgie betreut werden. 


\section{Univ.-Prof. Dr. Lars Christian Rump}

Lars Christian Rump wurde am 25. Mai 1959 in Peine geboren. Nach dem Grundwehrdienst studierte er bis 1984 Medizin an der Albert-Ludwigs-Universität Freiburg und wurde am Pharmakologischen Institut bei Univ.-Prof. Dr. Klaus Starke promoviert. Die experimentelle Arbeit zur Wirkung von $\alpha_{2}$-Adrenozeptorantagonisten auf die Nordrenalinfreisetzung wurde mit magna cum laude bewertet. 1984 bis 1985 war er Assistent bei Univ.-Prof. Dr. Robert Fischer an der Pathologie der Universität zu Köln und von 1985 bis 1987 als Feodor-Lynen-Stipendiat der Alexander von Humboldt-Stiftung am Department of Pharmacology der University of Melbourne, Australien. Hier beschäftigte er sich mit der neurohormonalen Regulation des renovaskulären Widerstandes und der Modulation von Neurotrans-

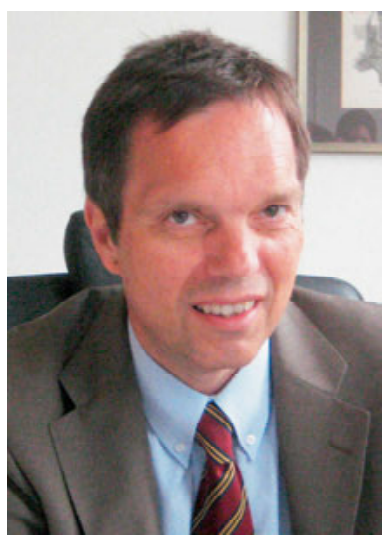
mittern durch das Renin-Angiotensin-System.

Ab 1987 erhielt Lars Christian Rump seine klinische Ausbildung zum Facharzt für Innere Medizin an der Universität Freiburg bei Univ.-Prof. Dr. Peter Schollmeyer. Nach der Habilitation 1993 zum Thema Modulation der renalen Neurotransmission durch präsynaptische Rezeptoren war er Oberarzt der Medizinischen Universitätsklinik Freiburg. Diese Tätigkeit wurde 1995 durch ein Stipendium der Deutschen Forschungsgemeinschaft (DFG) am Department of Pharmacology der University of California, San Diego bei Prof. Paul Insel unterbrochen. 1995 erhielt er den Nils-Alwall-Preis der Deutschen Arbeitsgemeinschaft für Klinische Nephrologie, 1996 den Schwerpunkt Nephrologie und 1999 die Ernennung zum außerplanmäßigen Professor.

Lars Christian Rump ist Hypertension Specialist der European Society of Hypertension, Hypertensiologe DHL (Deutsche Hochdruckliga) und Diabetologe DDG (Deutsche Diabetes Gesellschaft). 2002 nahm er den Ruf der Ruhr-Universität Bochum auf eine Professur für Innere Medizin (Schwerpunkt Nephrologie/Hypertonie) an und war Direktor der Medizinischen Klinik 1 am Marienhospital Herne. 2003 bis 2007 war er Studiendekan in Bochum.

Schwerpunkte seiner Forschung sind molekulare Mechanismen der Progression von Nierenerkrankungen, Hypertonie und kardiovaskuläre Komplikationen bei Niereninsuffizienz. Lars Christian Rump hat mehr als 125 pubmed-gelistete Publikationen verfasst und ist DFG-Gutachter für Sachmittelbeihilfen, Forschergruppen und Sonderforschungsbereiche. Er ist im Editorial Board mehrerer internationaler Fachzeitschriften und aktives Mitglied in zehn wissenschaftlichen Fachgesellschaften. Im Februar 2007 erhielt er den Ruf auf den Lehrstuhl für Innere Medizin (Schwerpunkt Nephrologie) und ist seit dem 1. Oktober 2007 Direktor der Klinik für Nephrologie am Universitätsklinikum der HeinrichHeine-Universität Düsseldorf. 


\section{Univ.-Prof. Dr. Alfons Schnitzler}

Alfons Schnitzler ist seit April 2008 Inhaber des Lehrstuhls für Klinische Neurowissenschaften und Medizinische Psychologie an der Heinrich-Heine-Universität und Direktor des gleichnamigen Instituts. Das Institut wird unter seiner Leitung eine neurowissenschaftliche Ausrichtung einnehmen. Er ist gleichzeitig Ärztlicher Leiter des Zentrums für Bewegungsstörungen und Neuromodulation der Neurologischen Klinik. In diesem Bereich ist auch die Tiefe Hirnstimulation angesiedelt, die in Düsseldorf von Neurologie und Neurochirurgie gemeinsam bei Patienten mit Bewegungsstörungen, wie zum Beispiel der Parkinson'schen Krankheit, eingesetzt wird.

Alfons Schnitzler studierte von 1979 bis 1986 Humanmedizin an der RWTH Aachen, der Christian-Albrechts-

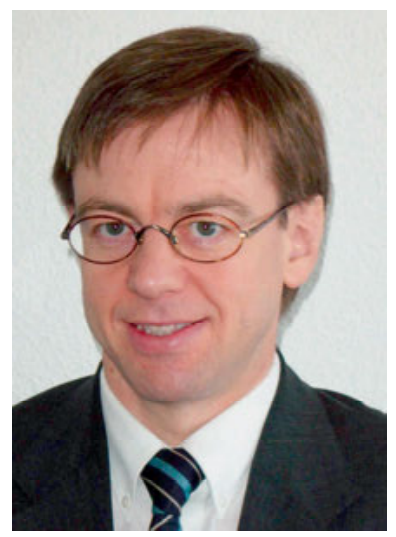
Universität Kiel und der University of Cambridge (Großbritannien). Er erhielt 1986 seine Approbation als Arzt und wurde über ein experimentelles neurophysiologisches Thema mit summa cum laude promoviert. Seine Habilitation und die Erteilung der Venia legendi für das Fach Neurologie und Neurophysiologie erfolgten im Jahr 1998.

Von 1986 bis 1987 war Alfons Schnitzler zunächst wissenschaftlicher Mitarbeiter der Abteilung Klinische Neuropharmakologie der Troponwerke Köln, dann Assistenzarzt und wissenschaftlicher Mitarbeiter an der Psychiatrischen Klinik der Heinrich-Heine-Universität und seit 1991 an der Neurologischen Klinik der Heinrich-Heine-Universität. Forschungsaufenthalte führten ihn in dieser Zeit nach Kanada und Finnland. Alfons Schnitzler ist seit 1993 Facharzt für Psychiatrie und seit 1995 für das Fach Neurologie.

Im Jahr 1998 wurde Alfons Schnitzler klinischer Oberarzt der Neurologischen Klinik und Leiter einer Magnetenzephalografie-Nachwuchsgruppe, seit 2001 leitet er den klinischen Schwerpunkt „Bewegungsstörungen und tiefe Hirnstimulation“ sowie das Regionalzentrum Düsseldorf im Kompetenznetz „Parkinson“ des Bundesministeriums für Bildung und Forschung. Er wurde 2003 außerplanmäßiger Professor an der Heinrich-Heine-Universität.

Im April 2006 war Alfons Schnitzler auf eine W2-Professur für Neurologie an der Heinrich-Heine-Universität berufen worden und hatte dafür einen Ruf auf einen Lehrstuhl für Cognitive Neuroscience an der Simon Fraser University in Vancouver, Kanada, abgelehnt. 2007 hatte er den Lehrstuhl für Neuroscience und Neurology am Wales Institute of Cognitive Neuroscience der Universität in Bangor, Wales, inne. Ebenfalls 2007 erhielt er einen Ruf auf den Lehrstuhl für Neurologie am Klinikum Bergmannsheil der Ruhr-Universität Bochum, entschied sich aber für das Angebot aus Düsseldorf. 


\section{Univ.-Prof. Dr. Ute Spiekerkötter}

Am 1. März 2008 wurde Ute Spiekerkötter zur W2-Professorin für Pädiatrische Stoffwechselstörungen ernannt und ist seitdem Leiterin des Bereichs Pädiatrische Stoffwechselstörungen in der Universitätskinderklinik.

Sie wurde 1965 in Herne geboren und studierte von 1986 bis 1992 Humanmedizin an den Universitäten Aachen und Göttingen. Das Praktische Jahr absolvierte sie an den Universitäten Münster und Kathmandu, Nepal. Während und nach dem Studium war Ute Spiekerkötter in verschiedenen Entwicklungshilfeprojekten unter anderem mit den Ärzten für die Dritte Welt in Indien und Zimbabwe tätig. 1995 wurde Ute Spiekerkötter mit der Arbeit Phenobarbital-Entzug bei epilepsiekranken Kindern promoviert.

Ihre Weiterbildung zur Fachärztin für Kinderheilkunde

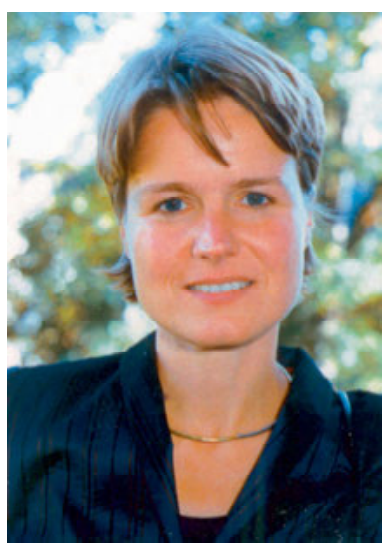
absolvierte Ute Spiekerkötter von 1994 bis 2000 an den Universitäten Essen und Düsseldorf. Im Jahr 2000 erhielt sie die Facharztanerkennung für das Fach Kinderheilkunde.

Von 2001 bis 2003 arbeitete Ute Spiekerkötter im Rahmen eines postdoctoral fellowship am Children's Hospital der Vanderbilt University in Nashville, Tennessee, USA. 2003 kehrte sie an die Universitätskinderklinik Düsseldorf zurück.

Im Jahre 2004 wurde Ute Spiekerkötter durch den Hendrik Casimir-Karl Ziegler-Forschungspreis der Nordrhein-Westfälischen Akademie der Wissenschaften und der Königlich Niederländischen Akademie der Wissenschaften ausgezeichnet, der ihr von 2004 bis 2005 ein zweites postdoctoral fellowship an der Universität von Amsterdam, Niederlande, ermöglichte.

Seit 2005 ist Ute Spiekerkötter als Oberärztin in der Klinik für Allgemeine Pädiatrie an der Universitätskinderklinik Düsseldorf tätig. Im selben Jahr habilitierte sie sich mit dem Thema: Angeborene Störungen der Oxidation Langkettiger Fettsäuren - Molekulare und Pathophysiologische Untersuchungen und erhielt die Venia legendi für das Fach Kinderheilkunde.

Ute Spiekerkötter leitet eine wissenschaftliche Arbeitsgruppe, die die Pathomechanismen bei Störungen der Fettsäurenoxidation untersucht. Ihre Arbeiten sind gefördert durch die Deutsche Forschungsgemeinschaft und den Sonderforschungsbereich 575.

Ute Spiekerkötter ist verheiratet und hat eine Tochter. 


\section{Univ.-Prof. Dr. Sascha Weggen}

Sascha Weggen, geboren 1970 in Kassel, studierte Biologie an den Universitäten Aachen und Bonn und wurde 1999 am Institut für Neuropathologie der Rheinischen Friedrich-Wilhelms-Universität Bonn in der Arbeitsgruppe von Univ.-Prof. Dr. Otmar Wiestler mit Studien zur Entstehung kindlicher Gehirntumoren promoviert.

Zwischen 2000 und 2004 arbeitete er, gefördert durch das Emmy Noether-Programm der Deutschen Forschungsgemeinschaft (DFG), als Postdoktorand in der Arbeitsgruppe von Prof. Edward Koo an der University of California, San Diego. Dort begann er, sich mit Therapieansätzen für die Alzheimer-Erkrankung, die häufigste altersabhängige neurodegenerative Erkrankung mit etwa fünf Millionen Patienten in der Europäischen Region, zu beschäfti-

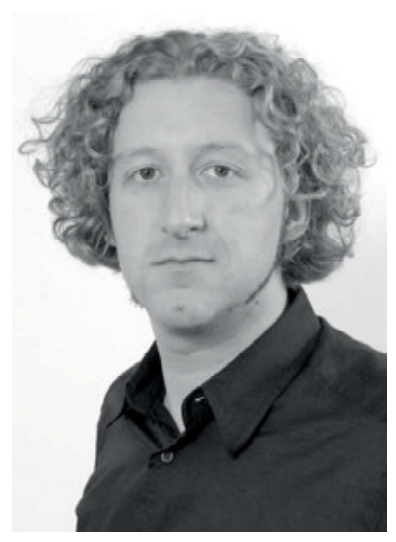
gen. In dieser Zeit entdeckte er eine neue Klasse von chemischen Substanzen, die mit hoher Spezifität die Bildung des A $\beta 42$-Peptides, eines Proteinfragmentes, unterdrücken. Genetische, tierexperimentelle und toxikologische Studien belegen, dass eine verstärkte

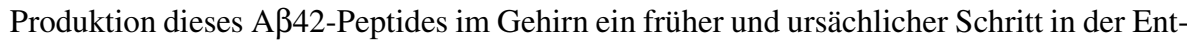
wicklung der Alzheimer-Erkrankung ist. Ein Molekül dieser Substanzklasse wird zurzeit in klinischen Phase-III-Studien auf Wirksamkeit bei Alzheimer-Patienten getestet.

Nach seiner Rückkehr aus den USA leitete er zwischen 2004 und 2007 eine Emmy Noether-Arbeitsgruppe der DFG am Institut für Physiologische Chemie und Pathobiochemie an der Johannes-Gutenberg-Universität Mainz. Im Mai 2007 nahm Sascha Weggen den Ruf auf die Professur „Molekulare Neuropathologie“ am Institut für Neuropathologie der Heinrich-Heine-Universität Düsseldorf an. Seit 2008 ist er Koordinator eines Verbundprojektes im „Kompetenznetz Degenerative Demenzen“ (KNDD), einem bundesweiten Forschungsnetzwerk, das grundlagenwissenschaftliche und klinische Fragestellungen bei Demenzerkrankungen bearbeitet und durch das Bundesministerium für Bildung und Forschung (BMBF) gefördert wird.

Die derzeitigen Forschungsschwerpunkte seiner Arbeitsgruppe sind Strategien zur Therapie und Prävention der Alzheimer-Erkrankung, die Ursachen der familiären Formen der Alzheimer-Erkrankung sowie die Rolle von Entzündungsprozessen bei neurodegenerativen Erkrankungen. 


\section{Univ.-Prof. Dr. Wolfgang Wölwer}

Wolfgang Wölwer studierte von 1978 bis 1983 Psychologie an der Universität Düsseldorf und erwarb dort sein Diplom mit einer Abschlussarbeit zur Wirkung von Benzodiazepin-Tranquilizern auf emotionale Prozesse. Anschließend arbeitete er bis 1988 als wissenschaftlicher Mitarbeiter in der Abteilung Physiologische Psychologie der Technischen Universität Berlin, an der er mit experimentellen Untersuchungen zu neuropsychologischen Modellvorstellungen und Verhaltensindikatoren von Angst promoviert wurde. Von 1988 bis 1992 war er wissenschaftlicher Mitarbeiter an der Psychiatrischen Klinik und Poliklinik der Freien Universität Berlin.

1992 wechselte Wolfgang Wölwer an die Rheinischen Kliniken Düsseldorf, Klinik und Poliklinik für Psychiatrie und Psychotherapie der Heinrich-Heine-Universität, an der

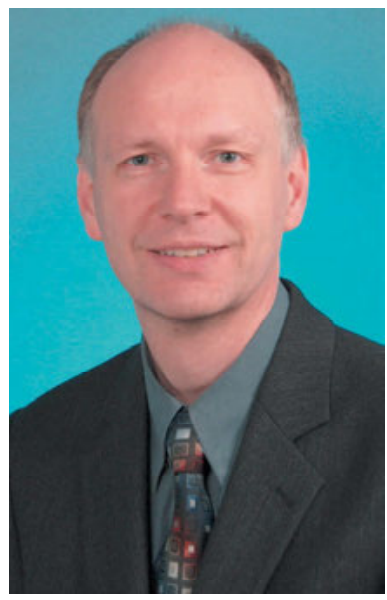
er die Leitung des Forschungslabors für Experimentelle Psychopathologie sowie die Koleitung einer Forschungsgruppe zu Abhängigkeitserkrankungen übernahm. Seit 1999 leitet er zudem die Netzwerkzentrale des vom Bundesministerium für Bildung und Forschung (BMBF) geförderten bundesweiten Forschungsverbunds Kompetenznetz Schizophrenie, in dessen Konzeption und Beantragung er zuvor schon wesentlich involviert war. Im Jahr 1999 erhielt er die Approbation als Psychologischer Psychotherapeut. 2005 habilitierte er sich mit Untersuchungen zur Differenzierung kognitiver Störungen schizophren Erkrankter mittels Augenbewegungsanalysen für das Fach Medizinische Psychologie an der Heinrich-Heine-Universität Düsseldorf, von der er im Jahr 2007 einen Ruf auf die neu geschaffene W2-Professur für Experimentelle Psychopathologie erhielt. Diese soll den Schwerpunkt Schizophrenieforschung an der Klinik und Poliklinik für Psychiatrie und Psychotherapie stärken und beinhaltet weiterhin die Leitung der Netzwerkzentrale des Kompetenznetzes Schizophrenie. Die Ernennung erfolgte zum 1. Juni 2008.

Schwerpunkte der wissenschaftlichen Arbeit von Wolfgang Wölwer sind die Erforschung kognitiver und emotionaler Störungen schizophren Kranker sowie die Entwicklung und Evaluation verhaltenstherapeutischer Ansätze bei schizophren Erkrankten und Alkoholabhängigen. Besondere Aufmerksamkeit widmet er Beeinträchtigungen sozial-kognitiver Prozesse bei schizophrenen Störungen, für die er mit seiner Forschungsgruppe neue rehabilitative Ansätze entwickelt hat. Seine bisherigen Forschungsarbeiten wurden wesentlich vom BMBF und der Deutschen Forschungsgemeinschaft gefördert. 
